\title{
RESPUESTA A LA IDEBENONA ASOCIADA A MULTIVITAMINOTERAPIA EN NEUROPATÍA ÓPTICA HEREDITARIA DE LEBER
}

\section{RESPONSE TO IDEBENONE AND MULTIVITAMIN THERAPY IN LEBER'S HEREDITARY OPTIC NEUROPATHY}

\author{
BARNILS N ${ }^{1}$, MESA E $^{1}$, MUNOOZ $\mathrm{S}^{1}$, FERRER-ARTOLA A ${ }^{2}$, ARRUGA J 3
}

\section{RESUMEN}

Objetivo: Determinar la eficacia del tratamiento con idebenona y multivitamínico en la neuropatía óptica hereditaria de Leber (NOHL).

Método: Dos pacientes diagnosticados de NOHL, fueron tratados con idebenona, vitamina $\mathrm{C}$ y riboflavina durante un año. Ambos fueron evaluados clínicamente antes, durante y después del tratamiento.

Resultado: Ninguno de los dos pacientes experimentó mejoría visual y ambos sufrieron afectación en el segundo ojo.

Conclusiones: A pesar de casos publicados en la literatura de recuperación visual con idebenona en pacientes con NOHL, nuestra experiencia indica que este tratamiento no es efectivo para la enfermedad de Leber.

Palabras clave: Idebenona, multivitaminas, neuropatía óptica hereditaria de Leber.

\section{ABSTRACT}

Objective: To ascertain the efficacy of idebenone and multivitamin treatment in Leber's hereditary optic neuropathy (LHON).

Method: Two patients diagnosed of unilateral LHON were treated with megadoses of idebenone, vitamin $\mathrm{C}$ and riboflavin for one year. They were examined clinically before, during and after treatment.

Results: No improvement of visual function was observed. Despite the idebenone treatment, in both cases the second eye became involved.

Conclusions: Despite previous reports of visual recovery with idebenone in patients with LHON, our experience shows that an effective treatment for Leber's disease remains to be found (Arch Soc Esp Oftalmol 2007; 82: 377-380).

Key words: Idebenone, multivitamins, Leber's hereditary optic neuropathy, hereditary neuropathy.

\footnotetext{
Recibido: 22/5/06. Aceptado: 19/4/07.

Hospital Universitario de Bellvitge. L'Hospitalet de Llobregat. Barcelona. España.

1 Licenciado en Medicina.

2 Licenciado en Farmacia.

3 Doctor en Medicina.

Correspondencia:

Noemí Barnils García

Hospital Universitario de Bellvitge

Feixa Llarga, s/n

08907 L'Hospitalet de Llobregat (Barcelona)

España

E-mail: noebg76@hotmail.com
} 


\section{INTRODUCCIÓN}

La neuropatía óptica hereditaria de Leber (NOHL) es una enfermedad producida por mutación del ADN mitocondrial (ADNmt). Afecta predominantemente a varones y suele debutar entre los 15 y 35 años con una pérdida visual central e indolora unilateral, y afectación del segundo ojo en semanas o meses, a veces asociada a alteraciones neurológicas. En el fondo de ojo (FO) se observa una microangiopatía telangiectásica circumpapilar y una tumefacción de la capa de fibras nerviosas peripapilar, con evolución posterior a la atrofia óptica. No se dispone hasta la fecha de un tratamiento eficazi, aunque se han postulado diversas posibilidades. Se presentan dos pacientes afectos de NOHL, que fueron tratados con idebenona y vitaminas.

\section{CASOS CLÍNICOS}

\section{Caso 1}

Hombre de 30 años que consultó en marzo de 2001 por pérdida de visión en su ojo derecho (OD) de 10 días de evolución. La agudeza visual (AV) era contar dedos a $50 \mathrm{~cm}$ en OD y 1 en ojo izquierdo (OI), con abolición de la visión cromática y defecto pupilar aferente relativo (DPAR) en OD. En el FO vemos (fig. 1). Los resultados de los análisis bioquímicos, hematológicos y serológicos, y de la resonancia magnética (RM) cerebral no fueron relevantes. El estudio de ADNmt detectó la mutación G11778A en homoplasmia. Se administró megadosis de metilprednisolona (succinato sódico de metilprednisolona. Polvo para inyección. Inyectable. Frasco ampolla de $8 \mathrm{ml}$ a $14 \mathrm{ml}$ con diluente adjunto con conservante. Laboratorios Sanofi Aventis.

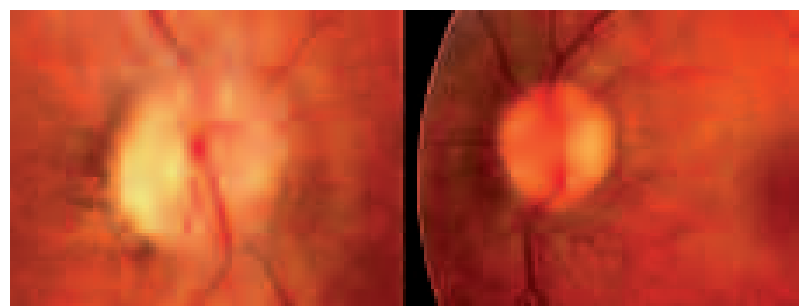

Fig. 1: Tumefacción de fibras nerviosas en papila de OD con palidez de zona temporal. Papila de OI de aspecto normal (caso 1).
SAU. Barcelona). posteriormente idebenona [6(hidroxi-decil-ubiquinona), Laboratorios Takeda Italia Farmaceutici, SpA, Rome, Italia) 270 mg/d, vitamina C (ácido ascórbico, Redoxon, contiene comprimidos con 500 mg, Laboratorios Bayer Hispania, Barcelona) $700 \mathrm{mg} / \mathrm{d}$ y riboflavina (becozyme $\mathrm{C}$ forte, compuesto multivitamínico contiene tiamina $15 \mathrm{mg}$, riboflavina $15 \mathrm{mg}$, nicotinamida $50 \mathrm{mg}$, piridoxina $10 \mathrm{mg}$, panteato de calcio $25 \mathrm{mg}$, biotina 150 $\mathrm{mg}$, cianocobalamina $10 \mathrm{mg}$, ácido ascórbico 200 mg, Laboratorios Bayer Hispania, Barcelona] 15 $\mathrm{mg} / \mathrm{d}$, durante 1 año. A los 11 meses de iniciado el tratamiento el paciente experimentó pérdida visual en su OI que ha permanecido estable hasta la actualidad, con AV de movimientos de manos OD y contar dedos a $20 \mathrm{~cm}$. en OI. FO (fig. 2).

\section{Caso 2}

Mujer de 19 años con antecedentes familiares de NOHL que consultó en octubre de 2003 por pérdida de visión en su OI de 4 días de evolución. La AV era de 1 en OD y contar dedos a $40 \mathrm{~cm}$ en OI, con disminución de la visión cromática y DPAR en OI.

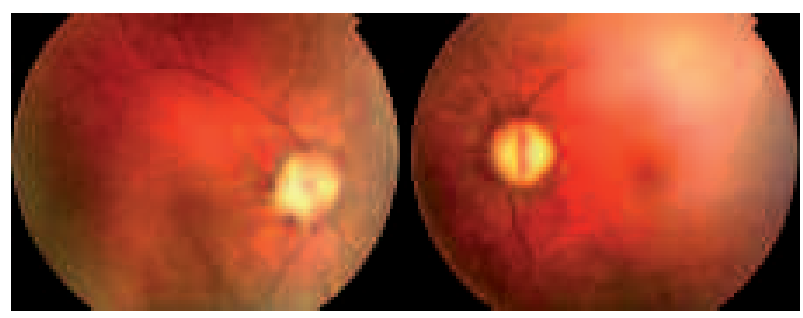

Fig. 2: Atrofia óptica bilateral (caso 1).

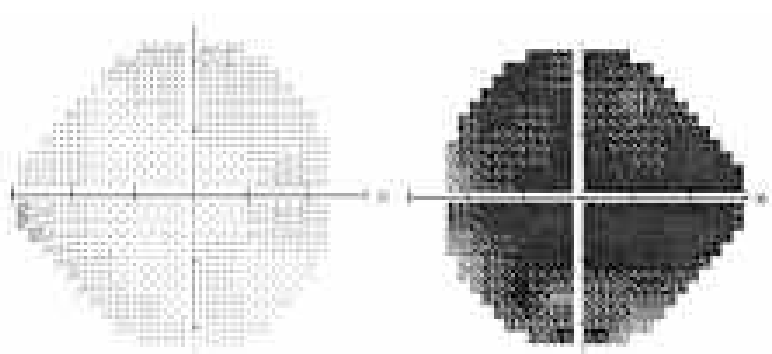

Fig. 3: Escala de grises de campo visual de Humphrey 30-2, OD: reducción de la sensibilidad periférica; OI: defecto global del campo con mayor densidad central (caso 2). 


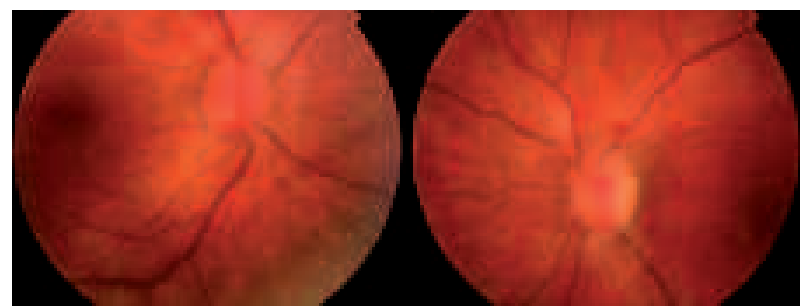

Fig. 4: Microangiopatía papilar en OD y tumefacción de papila OI con discreta palidez temporal y telangiectasias (caso 2).

Campo visual (fig. 3). FO (fig. 4). La AGF demostró ausencia de fuga del contraste desde el disco óptico, los resultados de los análisis bioquímicos, hematológicos y serológicos fueron normales y la RM cerebral reveló un aumento de la señal y una disminución del grosor del nervio óptico izquierdo. El estudio de ADNmt detectó la mutación G11778A en homoplasmia. Se administró megadosis de metilprednisolona y posteriormente idebenona $270 \mathrm{mg} / \mathrm{d}$, vitamina C $860 \mathrm{mg} / \mathrm{d}$ y riboflavina 9,6 $\mathrm{mg} / \mathrm{d}$, durante 1 año. A los 4 meses de iniciado el tratamiento experimentó pérdida visual en su OI que ha permanecido estable hasta la actualidad, con AV de contar dedos ambos ojos. FO (fig. 5).

\section{DISCUSIÓN}

La pérdida visual producida por la NOHL presenta una tendencia a la estabilidad, aunque se han descrito casos de recuperación visual espontánea, relacionados con la edad de debut de la enfermedad y con el tipo de mutación. De las diferentes mutaciones implicadas, la 11778, presente en nuestros pacientes, es la de peor pronóstico. En ambos casos se constataron hallazgos característicos de NOHL en el fondo de ojo y la AGF permitió descartar un verdadero edema de papila en la paciente 2 . No hay hallazgos en la tomografía computerizada (TC) o la

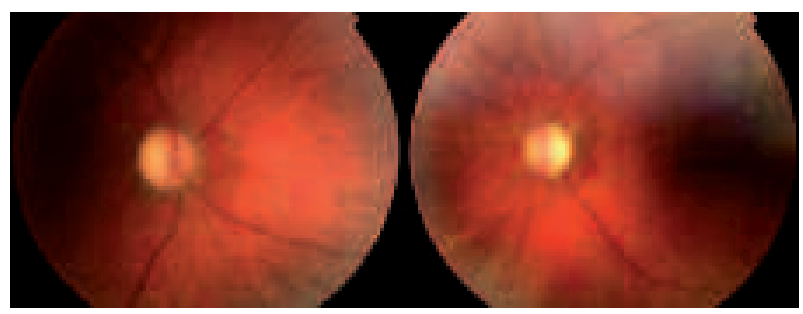

Fig. 5: Atrofia óptica bilateral (caso 2).
RM específicos de esta enfermedad, y las alteraciones aparecidas en la paciente 2 están en relación con la progresión hacia la atrofia óptica.

Han sido descritas alrededor de 15 mutaciones del ADNmt vinculadas a la NOHL, siendo las más frecuentes las mutaciones G11778A, G3460A y T14484C, llamadas primarias porque confieren el riesgo genético para la expresión individual de la NOHL (1). Ambos pacientes presentaban la 11778 en homoplasmia lo cual significa que todo su ADNmt se hallaba mutado. Considerando que algunas citopatías mitocondriales pueden responder a tratamientos que aumentan la producción de energía mitocondrial se ha probado el uso de la coenzima Q. En estos casos se ha utilizado la idebenona, una quinoleína precursora de la coenzima $Q$ que estimula la formación de ATP y actúa como un protector de la membrana mitocondrial contra la peroxidación lipídica $(2,3)$. Algunos autores han descrito una mejoría clínica tras este tratamiento, administrado solo o en combinación con vitaminas B2 y C (2-4). En 1992 Mashima publicó el caso de un paciente con NOHL, mutación 11778 en homoplasmia, que después de 7 meses de tratamiento con idebenona recuperó AV en ambos ojos (3). Otros dos autores, Cortelli (2) y Carelli (4), publicaron resultados similares añadiendo vitaminas, en sendos pacientes que tenían mutaciones 11778 y 14484, respectivamente, con recuperación, además, de síntomas neurológicos asociados. Por todos los hallazgos citados se ha sugerido que esta terapéutica podría acelerar o promover la recuperación visual de estos pacientes (5). En nuestros casos no se recuperó la visión y además se afectó su segundo ojo en el trascurso del tratamiento. No obstante, dado que existen casos de recuperación visual espontánea en pacientes con NOHL, no puede descartarse esta posibilidad en los casos publicados. En conclusión, nuestra experiencia no ha podido demostrar el éxito terapéutico del tratamiento con idebenona y multivitaminas.

\section{BIBLIOGRAFÍA}

1. Sánchez Dalmau B. Nunes V. Neuropatías ópticas heredodegenerativas. En: Arruga Ginebreda J. Sánchez Dalmau B. Neuropatías ópticas: diagnóstico y tratamiento. LXXVIII Ponencia oficial de la SEO. Madrid: Mac Line 2002; 143-147.

2. Cortelli P, Montagna P, Pierangeli G, Lodi R, Barboni P, Liguori $R$, et al. Clinical and brain bionergetics improvement with idebenone in a patient with Leber's hereditary 
optic neuropathy: a clinical and 31P-MRS study. J Neurol Sci 1997; 148: 25-31.

3. Mashima Y, Hiida Y, Oguchi Y. Remission of Leber's hereditary optic neuropathy with idebenone. Lancet 1992; 340: 368-369.

4. Carelli V, Barboni P, Zacchini A, Mancini R, Monari L, Cevoli S, et al. Leber's Hereditary Optic Neu- ropathy (LHON) with 14484/ND6 mutation in a North African patient. J Neurol Sci 1998; 160: 183 188.

5. Mashima Y, Kigasawa K, Wakakura M, Oguchi Y. Do idebenone and vitamin therapy shorten the time to achieve visual recovery in Leber hereditary optic neuropathy? J Neuroophthalmoly 2000; 20: 166-170. 